\title{
Laser-induced forward transfer on compliant receivers
}

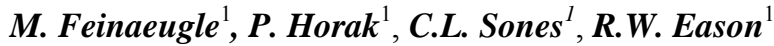 \\ 1. Optoelectronics Research Centre, University of Southampton, Highfield Campus, Southampton, SO17 1BJ, UK
}

Laser-induced forward transfer (LIFT) is a technique for the transfer of materials in solid or liquid phase. During LIFT a thin film (donor) previously coated onto a transparent carrier substrate is transferred by the explosive expansion of a small part of the donor volume after the absorption of a laser pulse at the interface of donor and carrier, accelerating a part of the thin film (flyer) towards a receiving substrate (receiver) [1]. When transferring a solid flyer via LIFT, it is possible to preserve its phase and physical properties, however such an intact transfer also depends strongly on the mechanical properties of the flyer and the receiver, and the flyer's velocity during transfer. For inelastic materials and high flyer velocities the resulting stresses on impact can exceed the flyer's mechanical strength and thus cause its undesirable shattering. To mitigate this effect, we have introduced a compliant polymer film capping the receiver and have studied experimentally the effect of such a film on the morphology and adhesion of a LIFTed deposit. Furthermore we modelled via finite element software (Comsol Multiphysics ${ }^{\circledR}$ ) the impact of a flyer onto such a receiver for different material parameters and transfer conditions, and compared it to the case of LIFT onto a bare glass receiver.

In the model, the impact forces and resulting stress on receiver and flyer were simulated for up to $1 \mathrm{~ms}$ after impact of the flyer on the receiver. Flyer velocity, polymer thickness, flyer geometry and Young's modulus were varied and the effect of interfacial stress on flyer and receiver were analysed. We could see that the introduction of a thin polymer reduced the stresses caused by impact of the flyer on the receiver.

For experimental comparison, donors were prepared from chalcogenide materials by sputtering thin films of bismuth (antimony) telluride onto fused silica carrier substrates. The LIFT setup consisted of a $\mathrm{KrF}$ excimer laser source emitting at $248 \mathrm{~nm}, 20 \mathrm{~ns}$ long pulses and a plano-convex lens that imaged a rectangular aperture with demagnification to the interface of donor and carrier. For the receivers, microscope slides were coated with thin polymer films and placed some microns away from the donor. We analysed the morphology and adhesion for LIFT as a function of incident laser fluence. Intact deposits with good adhesion were achieved for a receiver coated with a $10 \mu \mathrm{m}$ thick polydimethylsiloxane (PDMS) polymer as compared to both a receiver coated with other (thicker) polymers (SU-8 and Parafilm) and a bare glass receiver.
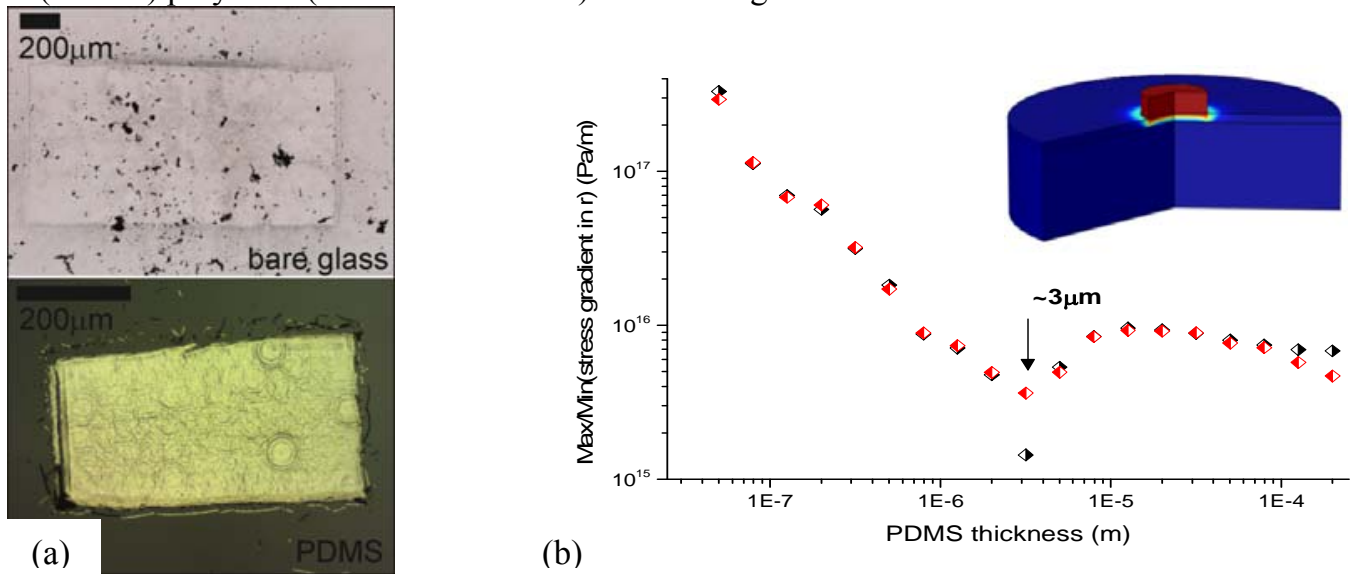

Fig. 1 (a) Experimental comparison of LIFTed deposit for glass receiver with and without PDMS coating for a bismuth telluride flyer. In the case of a bare glass receiver only debris is left while for a PDMS coated receiver the deposit is LIFTed intact. (b) The maximum modelled stress gradient in the radial direction is shown for a compliant PDMS layer with varying thickness. The ideal PDMS thickness is $\sim 3 \mu \mathrm{m}$. The inset shows a cut through a sketch of the modelled flyer/receiver combination at impact.

In conclusion we have shown the laser-induced forward transfer of $1 \mu \mathrm{m}$ thick chalcogenide flyers and the dependence of morphology and adhesion on the transfer and material parameters. The introduction of a thin polymer film improves the adhesion of the deposit and reduces the stresses during impact which can otherwise lead to the destruction of the flyer as shown in simulation and experiment.

\section{References}

[1] F. J. Adrian, J. Bohandy, B. F. Kim, A. N. Jette, and P. Thompson, "A study of the mechanism of metal deposition by the laser-induced forward transfer process,” Journal of Vacuum Science \& Technology B: Microelectronics and Nanometer Structures, 5, 5, 1490-1494, (1987). 